\title{
Discontinuous attractor dimension at the synchronization transition of time-delayed chaotic systems
}

\author{
Steffen Zeeb, ${ }^{1, *}$ Thomas Dahms, ${ }^{2}$ Valentin Flunkert, ${ }^{2,3}$ Eckehard Schöll, ${ }^{2}$ Ido Kanter, ${ }^{4}$ and Wolfgang Kinzel ${ }^{1}$ \\ ${ }^{1}$ Institute of Theoretical Physics, University of Würzburg, Am Hubland, D-97074 Würzburg, Germany \\ ${ }^{2}$ Institute of Theoretical Physics, Technical University of Berlin, Hardenbergstraße 36, D-10623 Berlin, Germany \\ ${ }^{3}$ Instituto de Fisica Interdisciplinar y Sistemas Complejos, IFISC (UIB-CSIC), Campus Universitat de les Illes Balears, \\ E-07122 Palma de Mallorca, Spain \\ ${ }^{4}$ Department of Physics, Bar-Ilan University, Ramat-Gan 52900, Israel
}

(Received 11 October 2012; revised manuscript received 14 January 2013; published 10 April 2013)

\begin{abstract}
The attractor dimension at the transition to complete synchronization in a network of chaotic units with timedelayed couplings is investigated. In particular, we determine the Kaplan-Yorke dimension from the spectrum of Lyapunov exponents for iterated maps and for two coupled semiconductor lasers. We argue that the Kaplan-Yorke dimension must be discontinuous at the transition and compare it to the correlation dimension. For a system of Bernoulli maps, we indeed find a jump in the correlation dimension. The magnitude of the discontinuity in the Kaplan-Yorke dimension is calculated for networks of Bernoulli units as a function of the network size. Furthermore, the scaling of the Kaplan-Yorke dimension as well as of the Kolmogorov entropy with system size and time delay is investigated.
\end{abstract}

DOI: 10.1103/PhysRevE.87.042910

PACS number(s): 05.45.Pq, 02.30.Ks

\section{INTRODUCTION}

Networks of identical nonlinear units which are coupled by their dynamic variables can synchronize to a common chaotic trajectory $[1,2]$. This phenomenon is of fundamental interest in nonlinear dynamics, with applications in neural networks, coupled lasers, electronic networks, and secure communication [3-6]. For many applications, the coupling signals are transmitted with a time delay which is much larger than the internal time scales of the individual units. A network of identical units with identical delay times can synchronize to a common chaotic trajectory without any time shift between these units (zero lag synchronization) [7]. This can only occur for so-called weak chaos [8], i.e., if the largest Lyapunov exponent (LE) of the network decays with the inverse delay time. More general systems with multiple delays are also able to synchronize completely [9-12].

A chaotic system with time-delayed couplings (including self-feedback for a single unit) has special mathematical properties. The system becomes infinite dimensional and has a continuous spectrum of LEs. The dimension of the chaotic attractor increases proportional to the delay time [13,14]. In this contribution, we investigate the attractor dimension close to the transition to chaos synchronization.

Various systems display a change or discontinuity in their statistical properties, e.g., information transfer and statistical complexity, at the emergence of collective behavior such as the transition to chaos synchronization [15]. In fact, chaos synchronization requires contracting directions in phase space [16]. However, typically the LEs are continuous when crossing the transition to synchronization. Hence quantities derived from the spectrum of LEs seem to be continuous as well. However, in this paper, we show that the attractor dimension of a chaotic system with long delay times is

*steffen.zeeb@physik.uni-wuerzburg.de discontinuous at the transition to synchronization. This is a consequence of a dense spectrum of LEs perpendicular to the synchronization manifold due to the delayed feedback and coupling.

The dimension of a chaotic attractor is not uniquely defined [17]. We consider two attractor dimensions: the Kaplan-Yorke (KY) dimension [18] $D_{K Y}$, which is determined from the spectrum of the LEs, and the correlation dimension $D_{C}$ [19]. For typical attractors, the KY dimension is conjectured to be identical to the information dimension $D_{I}$. This is known as the so-called KY conjecture $[17,20]$. The information dimension, in turn, is an upper bound for the correlation dimension, $D_{I} \geqslant D_{C}[21]$. Note that "typical" is used since there exist nongeneric examples for which the KY conjecture does not hold $[22,23]$. But the validity of the KY conjecture is generally restored when adding small perturbations to these nongeneric examples.

In this paper, we study these two attractor dimensions, $D_{K Y}$ and $D_{C}$, for networks of identical nonlinear units coupled by their time-delayed variables. The delay time is much larger than the internal time scales, and chaos is generated by the coupling. Increasing the coupling strength, the system has a transition to complete synchronization [16].

We show that the KY dimension is discontinuous at the synchronization transition; it jumps from a high value for the unsynchronized to a low value for the synchronized chaotic attractor. This is a general result which holds for any network with time-delayed couplings. We numerically calculate the KY dimension for networks of iterated maps and for two coupled semiconductor lasers. For networks of Bernoulli maps, we are also able to obtain analytical results. As a cross-check, the correlation dimension of the systems is computed as well and, (at least) for networks of Bernoulli maps, it displays a clear discontinuity at the synchronization transition, which is in agreement with the jump in the KY dimension. Finally, we calculate the jump in the KY dimension as a function of the size of the network. 


\section{DISCONTINUOUS KAPLAN-YORKE DIMENSION}

Let us consider a network of $N$ identical nonlinear units. Each unit $j=1, \ldots, N$ has a set of dynamic variables $x_{j}(t)$ which obey the following differential equations with timedelayed couplings:

$$
\dot{x}_{j}(t)=F\left[x_{j}(t)\right]+\sigma \sum_{k=1}^{N} G_{j k} H\left[x_{k}(t-\tau)\right] .
$$

The function $F$ describes the local dynamics of each unit, the function $H$ couples the time-delayed variables of the connected units, and the adjacency matrix $G_{j k}$ defines the graph of the network. We restrict this matrix to have only non-negative entries and a constant row sum $\sum_{k} G_{j k}=1$. Thus the eigenvalue of $G$ with the largest modulus is always $\gamma_{1}=1$. The parameter $\sigma$ determines the strength of the coupling and $\tau$ is the delay time of the coupling.

By construction, the synchronization manifold (SM) $x_{j}(t)=s(t)$ is a solution of this network given by the equation

$$
\dot{s}(t)=F[s(t)]+\sigma H[s(t-\tau)] .
$$

We consider only networks where this equation has chaotic solutions for sufficiently large values of $\sigma$, i.e., the dynamics on the SM is chaotic and has at least one positive LE. The stability of the SM can be determined using the master stability function [24]. An infinitesimal perturbation of the synchronized trajectory can be decomposed into eigenvectors of the coupling matrix $G$ with corresponding eigenvalues $\gamma_{k}$, $k=1, \ldots, N$. The amplitudes $\xi_{k}(t)$ of the perturbations along these eigenvectors follow the equation

$$
\dot{\xi}_{k}(t)=D F[s(t)] \xi_{k}(t)+\sigma \gamma_{k} D H[s(t-\tau)] \xi_{k}(t-\tau) .
$$

Hence the perturbations are governed by a linear differential equation with time-delayed feedback and time-dependent coefficients. For each eigenvalue $\gamma_{k}$ of $G$, this equation yields a whole spectrum of LEs. A perturbation corresponding to the largest eigenvalue, $\gamma_{1}=1$, describes a perturbation within the SM. Hence $\gamma_{1}$ is called transversal eigenvalue. In the chaotic regime, the linearized dynamics is unstable for $\gamma_{1}=1$, i.e., it has positive LEs. Any arbitrary perturbation has, in general, components in the other eigenmodes with eigenvalues $\gamma_{k}, k=$ $2, \ldots, N$. These are the so-called longitudinal eigenvalues. Thus the SM is stable if Eq. (3) yields only negative LEs for all transversal eigenvalues such that perturbations transversal to the SM are decaying exponentially fast.

We consider only networks where the delay time $\tau$ is much larger than any other time scale of the system [25]. In this case, the condition for stability of the SM is given by the following equation [8]:

$$
\left|\gamma_{2}\right|<\exp \left(-\lambda_{m} \tau\right)
$$

Hence the transversal eigenvalue of $G$ with the largest absolute value, $\left|\gamma_{2}\right|$, and the maximum (longitudinal) LE, $\lambda_{m}$, of a single unit with feedback, given by Eq. (2), determine the stability of complete chaos synchronization. Note that $\lambda_{m}$ depends on the coupling strength $\sigma$, therefore Eq. (4) determines the critical coupling strength $\sigma_{c}$ where chaos synchronization appears.

In this work, we change a control parameter of the system such that the system exhibits a transition from a synchronized

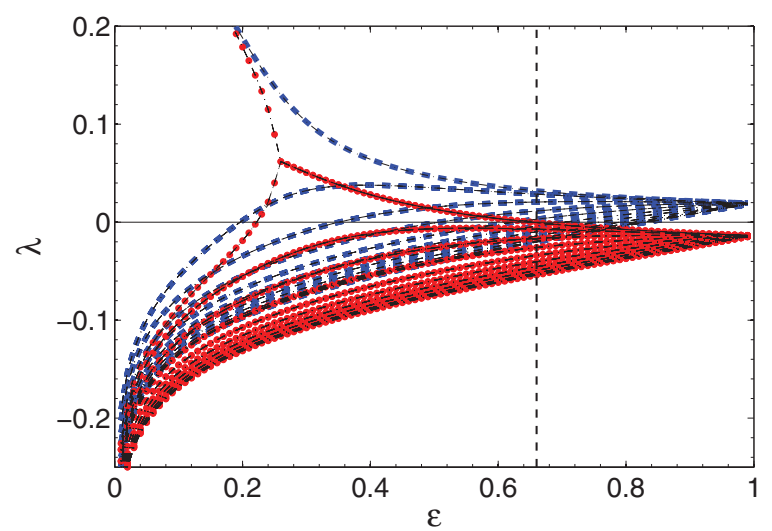

FIG. 1. (Color online) Lyapunov spectra vs coupling strength $\epsilon$ for a system of three all-to-all coupled Bernoulli maps with the parameters $a=1.5$ and $\tau=20$. The thick dashed blue lines show the longitudinal spectrum associated with $\gamma_{1}=1$ and the thick dotted red lines show the transversal spectrum associated with $\gamma_{2}=\gamma_{3}=-1 / 2$. Both spectra were obtained by solving the polynomial equations derived from the master stability function. The thin dash-dotted black lines show the Lyapunov spectrum computed by Gram-Schmidt from simulations of the full system. The vertical dashed line indicates $\epsilon_{c}$ where to its right the system is synchronized.

to an unsynchronized state. At this transition, we investigate the change in the attractor dimension.

A quantitative measure for the structure of the attractor is the KY dimension. It is defined by the spectrum of LEs obtained from Eq. (3). Considering a discrete spectrum $\lambda_{1} \geqslant \lambda_{2} \geqslant \ldots$, the KY dimension is defined as the largest number $M$ for which the sum of LEs is still positive plus an interpolation term which yields the fractal part of the dimension,

$$
D_{K Y}=M+\frac{\sum_{k=1}^{M} \lambda_{k}}{\left|\lambda_{M+1}\right|} .
$$

Now we want to show that the attractor dimension at the transition to chaos synchronization is discontinuous. The LEs are given by Eq. (3), where one obtains a whole spectrum of LE for each eigenvalue $\gamma_{k}$.

First, consider stable synchronization. For the longitudinal eigenvalue $\gamma_{1}=1$, the perturbations have, in general, positive as well as negative LEs. For all other (i.e., transversal) eigenvalues, the corresponding LEs are negative since the $\mathrm{SM}$ is stable. But close to the synchronization transition, the maximum LE of (at least) one of these transversal Lyapunov spectra approaches the value zero, as shown in Fig. 1. Consequently, close to the transition, these spectra contribute to the definition of the KY dimension, defined by Eq. (5).

However, this cannot be true. In the case of stable synchronization, the trajectory of the network, determined by Eq. (2), is completely restricted to the SM. Two neighboring trajectories inside the SM deviate from each other according to Eq. (3) with $\gamma_{1}=1$. Any transversal eigenvalues cannot contribute to the dynamics inside the SM. Thus, only the longitudinal LE spectrum can contribute to the KY dimension, i.e., the sum in Eq. (5) must only run over the LEs of the $\gamma_{1}$ spectrum.

Now, consider the dynamics outside but still close to the synchronization transition. In this case, the perturbations 
cannot be decomposed into the eigenmodes of the coupling matrix $G$ since the coefficients of the linearized equations of Eq. (1) depend on each single node. But close to a supercritical transition, we expect that the LEs are continuous as a function of $\sigma$ and the structure of the LEs is still similar to the one inside the SM, as is the case in Fig. 1.

In the desynchronized regime, all eigenvalues contribute to the KY dimension. Consequently, the KY dimension must be discontinuous at the transition to chaos synchronization. In fact, our results of Fig. 6 confirm this argument. The correlation dimension closely follows the KY dimension only if the transversal spectrum is omitted on the SM.

Chaos synchronization can only occur for weak chaos, where the largest LE scales with $1 / \tau$. In this case, the spectrum of LEs is dense. Hence, a large number of additional LEs contributes to the KY dimension at the transition, and we expect the jump of the KY dimension to be of the order of the size $N$ of the network.

From the Lyapunov spectrum, we can also calculate the Kolmogorov entropy, which quantifies the predictability of the system [26]. It is defined as the sum over all positive LEs,

$$
K=\sum_{i} \lambda_{i} \text { for } \lambda_{i}>0 .
$$

Since only positive LEs contribute, the entropy is always defined with the complete spectrum of LEs, hence it does not show a jump at the transition to chaos synchronization. Nevertheless, at the transition, at least one band of LEs adds to the entropy and we expect a discontinuous derivative of $K(\sigma)$.

The jump in the dimension of the chaotic attractor and the kink in the entropy are general results which should hold for any chaotic network at the transition to chaos synchronization. In the following section, we calculate the attractor dimensions $D_{K Y}$ and $D_{C}$ and the entropy $K$ for networks of iterated maps.

\section{ITERATED MAPS}

The previous general statement holds not only for differential equations, but also for networks of iterated maps with time-delayed coupling. Since such models are easier to analyze than continuous systems, we investigate networks of iterated maps in this section. Each unit $j$ has a one-dimensional variable in the unit interval $x_{t}^{j} \in[0,1]$, which is updated in discrete time steps $t$ according to the following equation:

$$
x_{t+1}^{j}=(1-\epsilon) f\left(x_{t}^{j}\right)+\epsilon \sum_{k} G_{j k} f\left(x_{t-\tau}^{k}\right) .
$$

The parameter $\epsilon$ is the coupling strength, but since the dynamic variable should stay in the unit interval, we subtract the nondelayed term with strength $\epsilon$. For the function $f(x)$, we use the Bernoulli shift and the asymmetric tent map.

Bernoulli map,

$$
x_{t+1}=\left(a x_{t}\right) \bmod 1 .
$$

Tent map,

$$
x_{t+1}= \begin{cases}\frac{1}{a} x_{t} & \text { for } \quad 0 \leqslant x_{t}<a \\ \frac{1}{1-a}\left(1-x_{t}\right) & \text { for } \quad a \leqslant x_{t} \leqslant 1 .\end{cases}
$$

The Bernoulli map is chaotic for a parameter $a>1$. For the chaotic tent map, the parameter $a$ is chosen such that the value $x_{t+1}$ stays in the range [0,1], i.e., $0<a<1$. For maps, we have a discrete delay time $\tau$. As before, the coupling matrix $G$ has constant row sum $\sum_{k} G_{j k}=1$.

The synchronized state is a solution of these equations and reads as

$$
s_{t+1}=(1-\epsilon) f\left(s_{t}\right)+\epsilon f\left(s_{t-\tau}\right) .
$$

As before, the perturbations of the SM can be associated with the eigenvalues $\gamma_{k}$ of the coupling matrix $G$. The amplitude $\xi_{t}^{k}$ of each mode obeys the linear master stability function determined by

$$
\xi_{t+1}^{k}=(1-\epsilon) f^{\prime}\left(s_{t}\right) \xi_{t}^{k}+\epsilon \gamma_{k} f^{\prime}\left(s_{t-\tau}\right) \xi_{t-\tau}^{k} .
$$

Note that for the Bernoulli network, the derivative $f^{\prime}=a$ is constant, therefore one only has to analyze linear equations with constant coefficients. For the tent map, however, the derivative can take on two different values and, hence, the coefficients change with time.

Since $\tau$ is discrete, for each mode with eigenvalue $\gamma_{k}$, one obtains $\tau+1$ many LEs. Figure 1 shows an example for a triangle of all-to-all coupled Bernoulli units with $\gamma_{1}=1$ and $\gamma_{2}=\gamma_{3}=-1 / 2$. For all values of $\epsilon$, the system is chaotic, since the largest LE of the $\gamma_{1}$ band is always positive. The transition to chaos synchronization occurs at $\epsilon_{c}$ where the maximum LE of the $\gamma_{2,3}$ band crosses the value zero.

The Lyapunov spectrum is, in general, obtained from a Gram-Schmidt orthonormalization procedure, according to Farmer [14]. The system's Eqs. (1) and (7), respectively, are linearized around the chaotic trajectory and simulated for a set of orthogonal perturbation vectors which have to be reorthogonalized after an appropriate amount of time. The Lyapunov spectrum is computed from the change in magnitude of the perturbation vectors.

For Bernoulli networks, we can also derive a polynomial equation of degree $\tau+1$ for the different eigenvalues $\gamma_{k}$ of the adjacency matrix $G$ from which the Lyapunov spectrum can easily be calculated. The polynomial equation reads as follows:

$$
z^{\tau+1}=(1-\epsilon) a z^{\tau}+\epsilon a \gamma_{k},
$$

where the LEs are given by $\lambda=\ln |z|$ [27]. This equation still holds in the desynchronized region since it does not depend on the trajectory of the system. Thus the LE spectrum can be calculated from Eqs. (9) and (10), respectively, in the complete parameter space. Note that both methods-orthonormalization procedure and polynomial equation-which compute the spectrum in completely different ways, yield the same results; see Fig. 1.

From Figs. 1 and 3, it can be seen that for $\epsilon$ values close to 1 , the LEs cluster into bands. This can be understood as follows. For $\epsilon=1$, the dynamical Eqs. (7) are given by

$$
x_{t+1}^{j}=\sum_{k=1}^{N} G_{j k} f\left(x_{t-\tau}^{k}\right) .
$$

Since the state at time $t+1$ is only influenced by the state at time $t-\tau$, the system is effectively given by $\tau+1$ uncoupled 
identical systems of the form

$$
\tilde{x}_{\theta+1}^{j}=\sum_{k} G_{j k} f\left(\tilde{x}_{\theta}^{k}\right) .
$$

Each of these $\tau+1$ systems is $N$ dimensional and gives rise to $N$ Lyapunov exponents. Since we have $\tau+1$ identical systems, each of these $N$ exponents is $\tau+1$ times degenerate. This holds as long as each effective system evolves on the same chaotic attractor, and thus does not rely on synchronization.

For $\epsilon<1$, the first term in Eq. (7) leads to a coupling between these effective systems and thus removes the degeneracy.

It is insightful to discuss this lifting of degeneracy for the case of the Bernoulli maps. For $\epsilon=1$, the solutions of the variational Eq. (10) are given by the complex $(\tau+1)$ th roots of $\gamma_{k} a$,

$$
z^{(0)}=\left(\gamma_{k} a\right)^{\frac{1}{\tau+1}} e^{i \frac{2 \pi}{\tau+1} l} \quad(l=0, \ldots, \tau),
$$

and the corresponding $(\tau+1)$ Lyapunov exponents are all equal and are given by

$$
\lambda^{(0)}=\ln \left|z^{(0)}\right|=\ln \left|\gamma_{k} a\right|^{\frac{1}{\tau+1}} .
$$

Here the superscript (0) indicates the zeroth order in an expansion in $1-\epsilon$.

For $\epsilon \neq 1$, we can make a perturbation expansion of Eq. (10) in the small parameter $\mu=1-\epsilon$. Inserting the ansatz $z=z^{(0)}+\mu \beta^{(1)}$, where $\beta^{(1)}$ is a coefficient that needs to be determined, into Eq. (10) yields the solutions up to first order in $\mu$,

$$
z^{(1)}=z^{(0)}+\mu \beta^{(1)}=z^{(0)}+\mu \frac{a}{\tau+1}\left[1-\frac{\gamma_{k}}{\left(z^{(0)}\right)^{\tau}}\right] .
$$

The values of the corresponding Lyapunov exponents $\left(\lambda^{(1)}=\right.$ $\left.\ln \left|z^{(1)}\right|\right)$ are depicted in Fig. 2 as a function of $\epsilon$. One clearly sees the lifting of degeneracy due to $\epsilon<1$.

Although for general maps $f$ one cannot write down an equation such as (10) in the unsynchronized regime, for $\epsilon=1$ the degeneracy follows rigorously from the discussion above, and for $\epsilon<1$ the interaction will generically lead to a lifting of degeneracy similar to that shown in Fig. 2.

In the synchronized regime, the linear Eqs. (3) and (9) can be used to compute the LE spectrum for any iterated map $f(x)$. Hence the LEs can be clustered into bands according

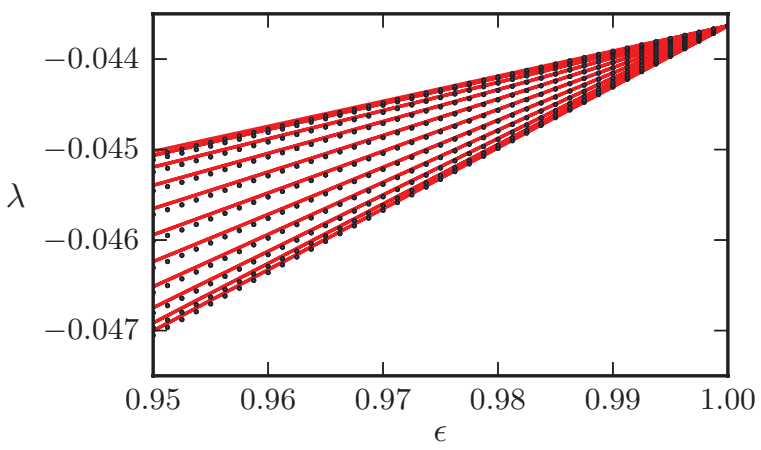

FIG. 2. (Color online) Perturbation expansion of Eq. (10) up to first order in $\mu=(1-\epsilon)$ (red lines) and exact location of eigenvalues (blue dots). Parameters: $a=0.4, \gamma_{k}=1, \tau=20$.

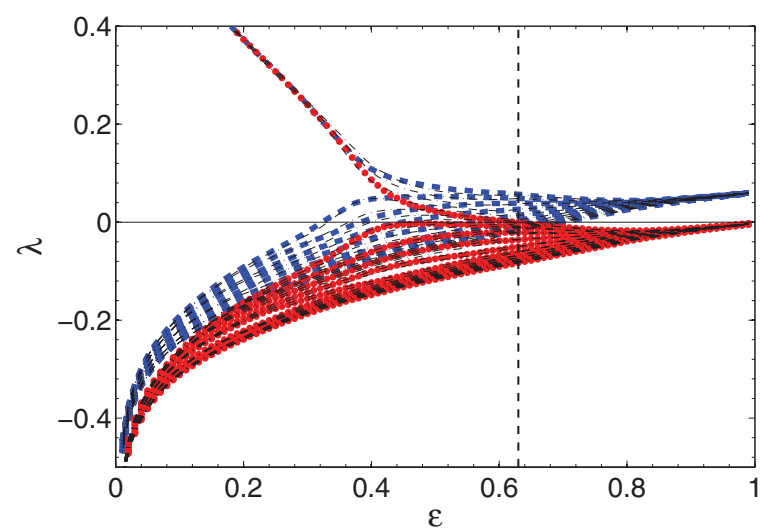

FIG. 3. (Color online) Lyapunov spectra vs coupling strength $\epsilon$ for a system of three all-to-all coupled tent maps with the parameters $a=0.4$ and $\tau=10$. The thick dashed blue lines show the longitudinal spectrum associated with $\gamma_{1}=1$ and the thick dotted red lines show the transversal spectrum associated with $\gamma_{2}=\gamma_{3}=-1 / 2$ computed by Gram-Schmidt from simulations of the master stability function. The thin dash-dotted black lines show the Lyapunov spectrum computed by Gram-Schmidt from simulations of the full system. The vertical dashed line indicates $\epsilon_{c}$ where to its right the system is synchronized. Note that for a better visibility, a system with $\tau=10$ is plotted.

to the eigenvalues of the coupling matrix $G$. However, for the unsynchronized system, these linear equations only hold for Bernoulli networks where the coefficients are constant and, in particular, independent of the systems trajectory. In general, in the desynchronized regime, we need to evaluate the linearized equations of the full system, i.e., Eqs. (1) and (7), to obtain the LE spectrum, and cannot restrict to the master stability function, i.e., Eqs. (3) and (9). But close to the transition we expect the spectra obtained from the master stability function to approximate the true spectra very well. Surprisingly, for the tent map, the results coincide very well not only close to the synchronizations transition, but for all values of $\epsilon$ when, for the desynchronized system in the master stability function given by Eq. (9), the dynamics of a single unit is inserted, e.g., $s_{t}$ and $s_{t-\tau}$ are replaced by $x_{t}^{1}$ and $x_{t-\tau}^{1}$, respectively. A comparison of the different spectra is shown in Fig. 3. In contrast to Fig. 1, the dashed blue and dotted red lines in Fig. 3 are obtained using the Gram-Schmidt procedure from simulating the master stability function, which is strictly only valid for the synchronized regime. The dash-dotted black lines are obtained using the Gram-Schmidt procedure on the full system's equations and therefor yields the correct results not only for the synchronized but also for the unsynchronized regime. Within the synchronized regime, the results match up to numerical accuracy, whereas outside of synchronization, the results of the two methods deviate since the master equation is no longer valid.

The KY dimension for the triangle of Bernoulli and tent maps, respectively, is shown in Fig. 4. The upper curve shows the KY dimension when the complete set of LEs is used in Eq. (5), while the lower curve uses only the LEs of the SM which are obtained from simulating a single unit. Note that an upper bound of the dimension is $3(\tau+1)$, i.e., the full system's 

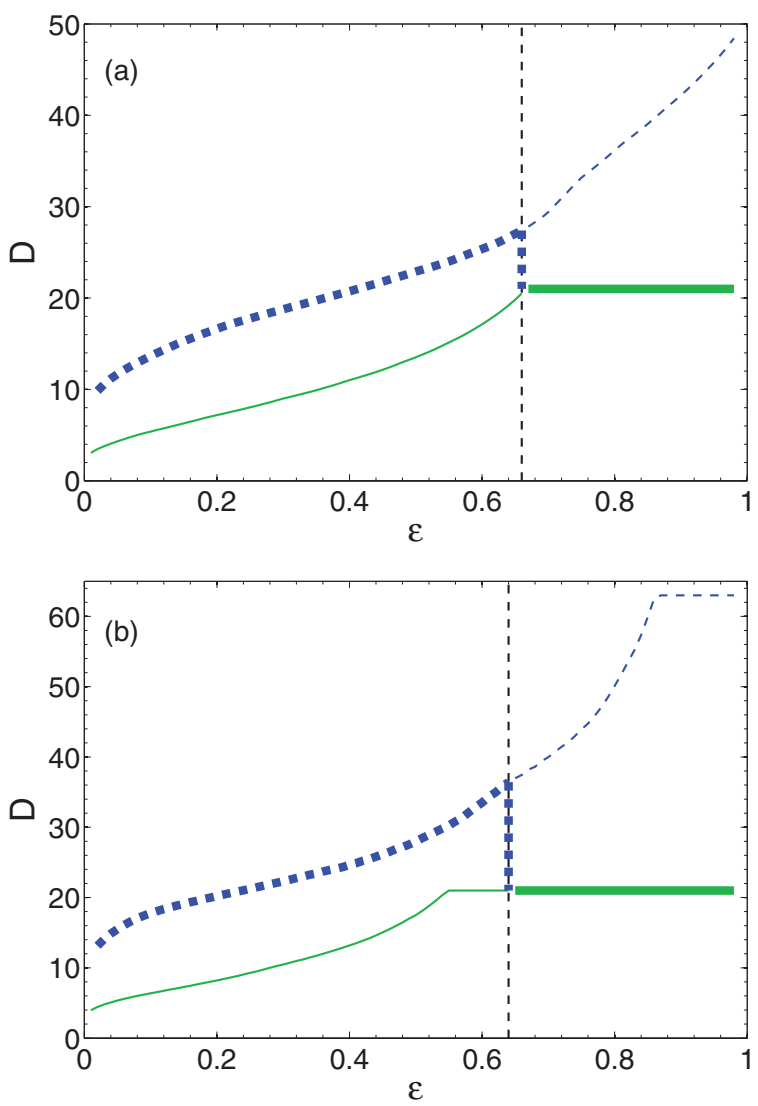

FIG. 4. (Color online) KY dimension $D_{K Y}$ with respect to the coupling strength $\epsilon$ for a system of three all-to-all coupled (a) Bernoulli and (b) tent maps, respectively. The upper blue dashed line shows $D_{K Y}$ of the full system; the lower green solid line shows $D_{K Y}$ of the SM. The vertical dashed line indicates $\epsilon_{c}$ where to its right the system is synchronized. The parameters are $\tau=20, a=1.5$ for the Bernoulli map and $a=0.4$ for the tent map, respectively.

dimension, for the desynchronized triangle and $\tau+1$ for the manifold.

As discussed in the previous section, in the synchronized region, the lower curve is valid, whereas in the desynchronized region, the upper curve is valid. Thus the KY dimension jumps to a lower value when the parameter $\epsilon$ is increased above $\epsilon_{c}$.

In the following, we consider a pair of maps with selffeedback. The dynamic equations of the system read

$x_{t+1}^{i}=(1-\epsilon) f\left(x_{t}^{i}\right)+\epsilon \kappa f\left(x_{t-\tau}^{i}\right)+\epsilon(1-\kappa) f\left(x_{t-\tau}^{j}\right)$,

with $i, j \in\{1,2\}$. The parameter $\epsilon$ is, as before, the coupling strength of the delayed terms to the internal dynamics and the parameter $\kappa$ determines the ratio between the external coupling and the self-feedback. The synchronized trajectory of the system also follows Eq. (8), which does not contain the strength $\kappa$ of the self-feedback. Hence the synchronized trajectory, i.e., the SM, is independent of $\kappa$ and only changes with the coupling strength $\epsilon$. Figure 6 shows the KY dimension as a function of $\kappa$. At the transition, the dimension jumps from the upper (dashed blue curve) to the lower constant value (solid green curve).

According to the discussion of the previous section, the KY dimension has to jump at the synchronization transition. This qualitative prediction of the KY conjecture should be valid for any measure of the dimension of the chaotic attractor. Thus, we also computed the correlation dimension of the system to compare it to the KY dimension and to check whether the dimension indeed jumps as we argue. For this purpose, we analyzed the system's trajectories, i.e., the time series of the system using the TISEAN package of Kantz and Schreiber [28]. In particular, the correlation function $C(\xi)$ according to Grassberger and Procaccia was computed, which scales as a power law $C(\xi) \propto \xi^{D_{C}}$ with the exponent being the correlation dimension $[19,22]$. A straight line was fitted to different correlation functions of different embedding dimensions in a double-logarithmic plot and, at the same time, the results were cross-checked in plots of the local slopes of the correlation function $d(\xi)=\partial C(\xi) / \partial \xi$ in which the power law behavior corresponds to a plateau. For more details on how to actually compute the correlation dimension, the reader is referred to $[28,29]$. Note that this method allows a reliable calculation of the correlation dimension for small values of the delay $\tau$ only.

A typical plot of the correlation functions as well as the local slopes is shown in Fig. 5. This figure shows that the extrapolation of the slope to low values of the radius $\xi$ is difficult. Hence our values for the correlation dimension give a lower bound to the actual correlation dimension since due to the limited
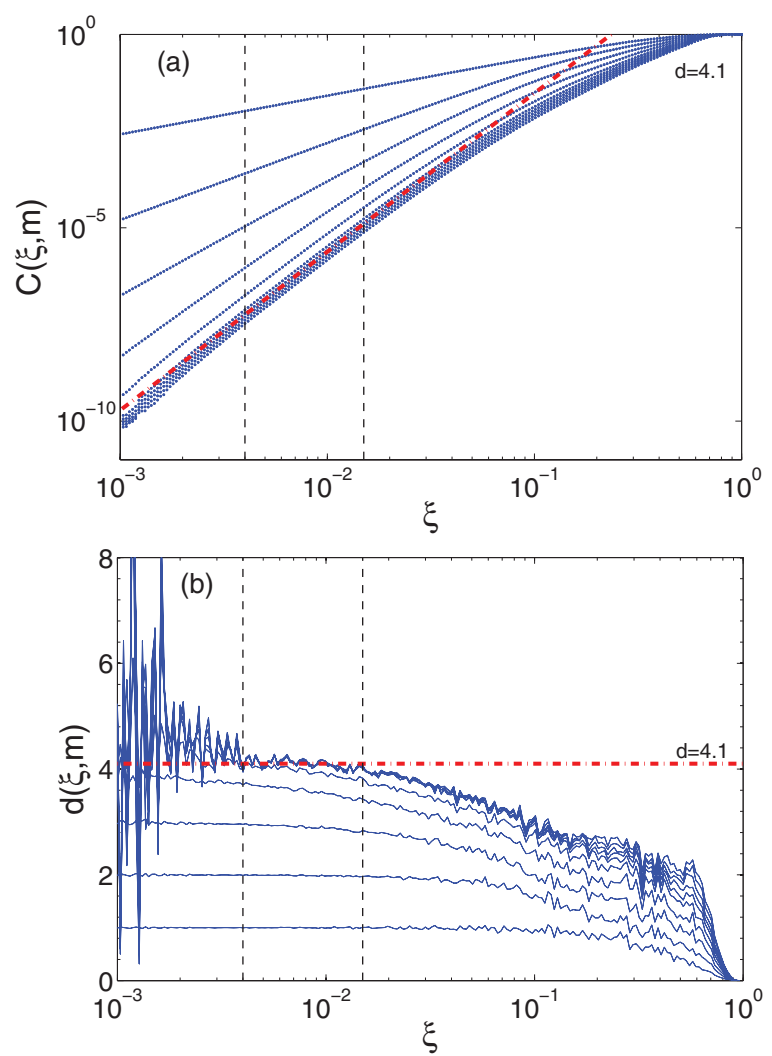

FIG. 5. (Color online) (a) Correlation function $C(\xi)$ and (b) local slope $d(\xi)$ for different embedding dimensions $m$ (different curves) computed from a time series of a system of two mutually coupled tent maps. The time delay is $\tau=5$, the length of the time series is $l=$ $10^{6}$, and the other parameters are $a=0.4, \epsilon=0.45$, and $\kappa=0.35$. Vertical dashed lines indicate the range that was used for the fit. The dash-dotted red line shows the fit. 

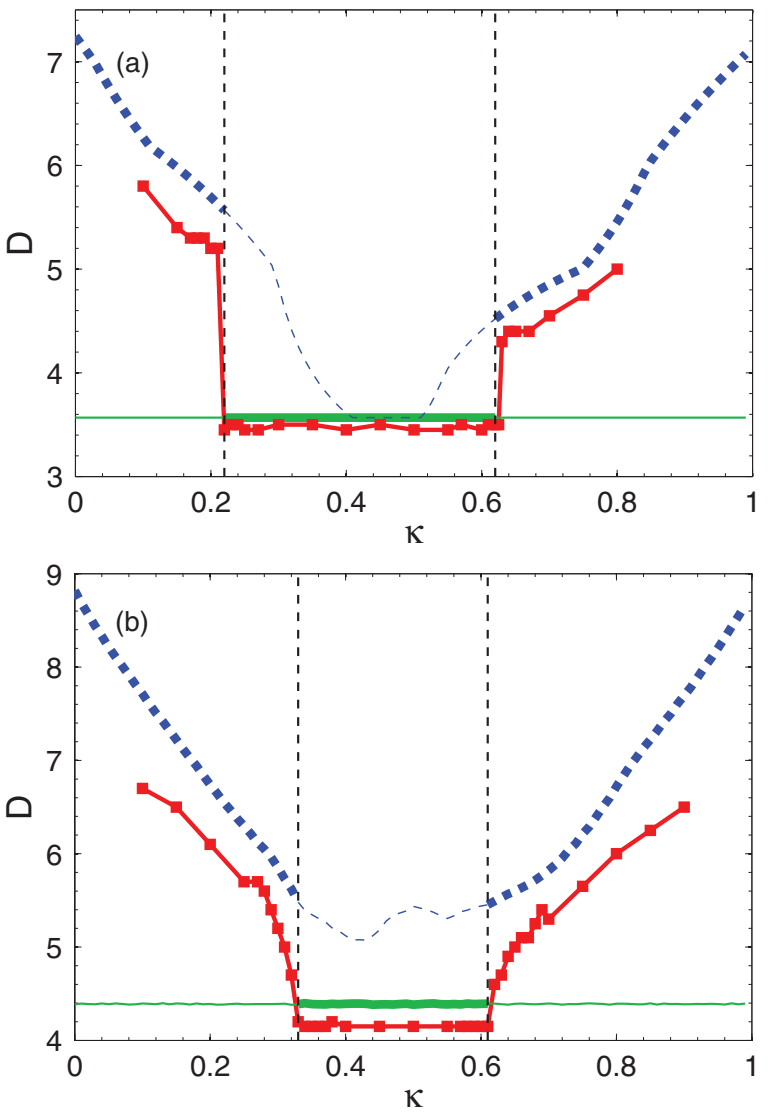

FIG. 6. (Color online) Attractor dimension vs coupling strength $\kappa$ for a system of two mutually coupled (a) Bernoulli and (b) tent maps, respectively. The time delay is $\tau=5$. The upper blue dashed line shows $D_{K Y}$ of the full system, the lower green solid line shows $D_{K Y}$ of the SM corresponding to $\gamma_{1}$, and the red curve in between shows $D_{C}$. The vertical dashed lines indicate $\kappa_{c}$. The parameters are $\tau=5, \epsilon=0.45, a=1.5$ for the Bernoulli map and $a=0.4$ for the tent map, respectively.

computational power it was not possible to analyze very small values of the distance $\xi$ with an appropriate accuracy.

Figure 6 shows the results for the KY and correlation dimension as a function of $\kappa$. The KY dimension is larger than the correlation dimension, which is in agreement with known theoretical inequalities. For the Bernoulli map, the correlation dimension displays a clear jump at the transition to synchronization, which is in agreement with our theoretical prediction. For the tent map, however, the discontinuity is not clearly visible from our results. As stated above, for small distances $\xi$, the correlation function $C(\xi)$ shows large fluctuations due to the limited statistics. From our results of Fig. 5, we cannot rule out that the local slopes might not be saturated yet. Thus the obtained results only serve as a lower bound which, according to our results, seems to increase with longer trajectories and better statistics. Consequently, the results of Fig. 6 do not rule out a discontinuous behavior of the attractor dimension. In any case, the synchronization transition is clearly visible in the discontinuous slope of the correlation dimension.

The Kolmogorov entropy, computed from Eq. (6), is shown for a pair of Bernoulli and tent maps, respectively, in Fig. 7.
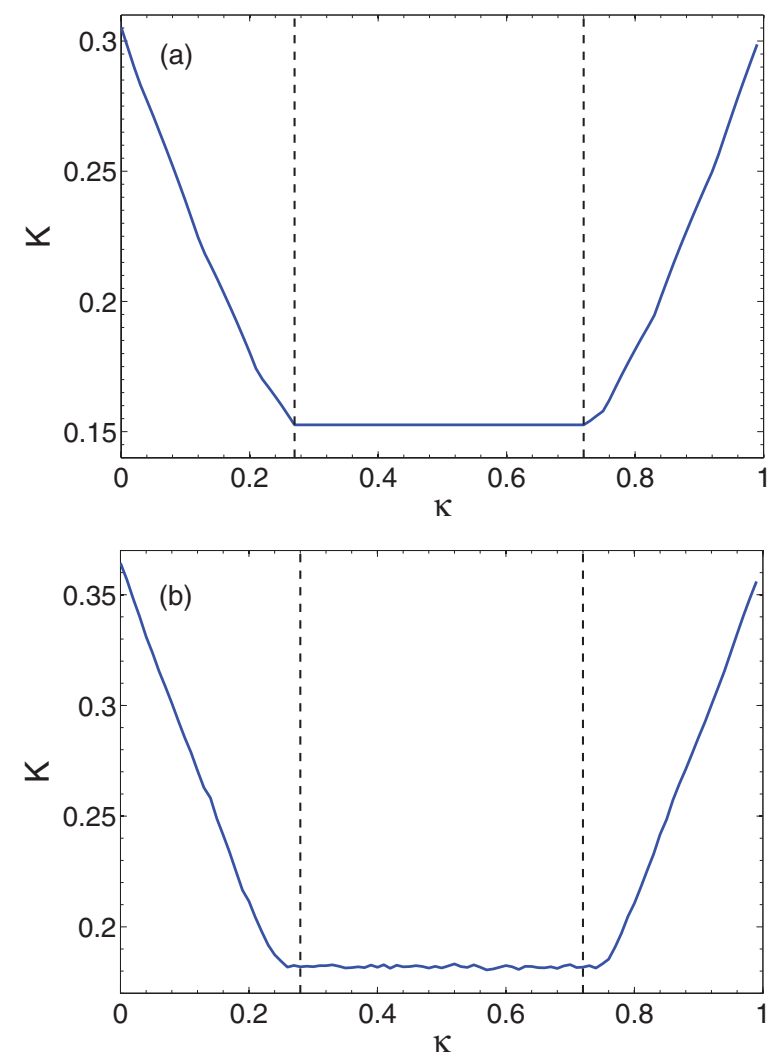

FIG. 7. (Color online) Kolmogorov entropy $K$ vs coupling strength $\kappa$ for a system of two mutually coupled (a) Bernoulli and (b) tent maps, respectively. The vertical dashed lines indicate $\kappa_{c}$. The parameters are $\tau=20, \epsilon=0.6, a=1.5$ for the Bernoulli map and $a=0.4$ for the tent map, respectively.

In the synchronized region, only the $\gamma_{1}$ band has positive LEs which contribute to the Kolmogorov entropy. At the synchronization transition, a kink in the entropy as a function of feedback strength can be seen when suddenly LEs from the other band contribute.

The result for the attractor dimensions and the prediction entropy are very similar for Bernoulli and tent maps. But we found a qualitative difference between the two models for the cross-correlations $C$ and the synchronization probability $\phi$ of a system of two mutually coupled units. The synchronization probability measures the fraction of time where the two trajectories are closer than some threshold $\Theta$ [30]. Figure 8 shows $C$ and $\phi$ with respect to the coupling strength $\kappa$ for fixed $\epsilon$. At the critical coupling $\kappa_{c}$, i.e., at the synchronization transition, both quantities $C$ and $\phi$ jump from a very low level to complete synchronization, $C=\phi=1$, for a system of Bernoulli maps, whereas for the tent map, $C$ and $\phi$ increase continuously to $C=\phi=1$.

The numerical results indicate that the synchronization transition for the tent map is of a supercritical type. That is, close to the transition to synchronization, there is a stable trajectory close to the SM and the dynamics is nearly synchronized. We thus observe a smooth transition to synchronization. In the Bernoulli map, on the other hand, the transition is of a subcritical type. Note that in both cases, a jump of KY dimension is predicted due to the bands of LEs. 

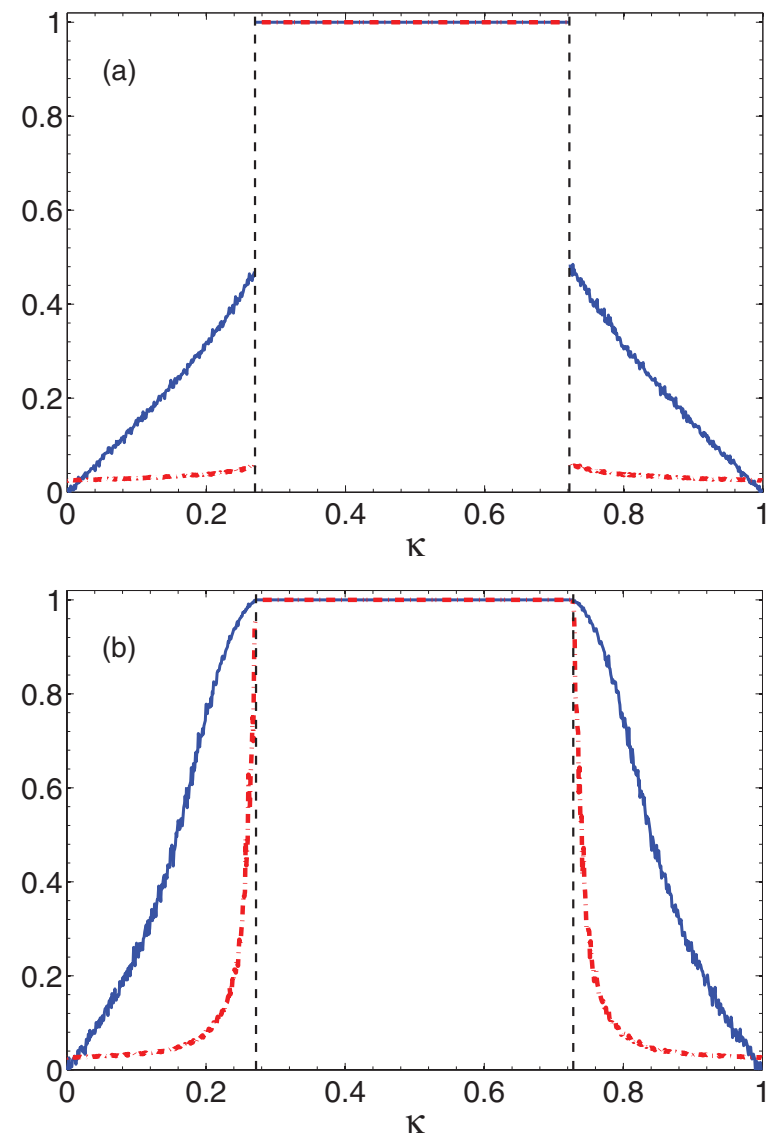

FIG. 8. (Color online) Cross-correlation $C$ (upper solid blue line) and synchronization probability $\phi$ (lower dashed red line) vs coupling strength $\kappa$ for a system of two mutually coupled (a) Bernoulli and (b) tent maps, respectively. The step size is $\Delta \kappa=10^{-3}$ and the other parameters are $\tau=20, \epsilon=0.6, a=1.5$ for the Bernoulli map and $a=0.4$ for the tent map, respectively. The threshold for $\phi$ was set to $\Theta=0.01$, i.e., the trajectories were assumed to be synchronized when they were closer together than $1 \%$ of their maximum distance.

\section{COUPLED LASERS}

An important application of Eq. (1) is the modeling of semiconductor lasers which are coupled by their mutual laser beams. To a good approximation, the dynamics of the laser intensity can be described by the Lang-Kobayashi rate equations [31]. The Lang-Kobayashi equations describe the dynamics of a laser with delayed feedback (or delayed coupling) in terms of a slowly varying complex electric field $E(t)$ and a population inversion $n(t)$. For our network of coupled lasers, the corresponding equations of the LangKobayashi type in dimensionless form are given by

$$
\begin{aligned}
\dot{E}^{i}(t) & =\frac{1}{2}(1+\mathrm{i} \alpha) n^{i}(t) E^{i}(t)+\sigma \sum_{j} G_{i j} E^{j}(t-\tau), \\
T \dot{n}^{i}(t) & =p-n^{i}(t)-\left[1+n^{i}(t)\right]\left|E^{i}(t)\right|^{2},
\end{aligned}
$$

where $E^{i}(t)$ is the envelope of the complex electric field and $n^{i}(t)$ is the renormalized population inversion of the charge carriers of laser $i$. The model parameters are summarized in Table I. The dimensionless delay time of $\tau=100$ translates to a delay time of the order of magnitude $1 \mathrm{~ns}$.
TABLE I. Parameters for the simulation of the Lang-Kobayashi equations.

\begin{tabular}{lcc}
\hline \hline Parameter & Symbol & Value \\
\hline Linewidth enhancement factor & $\alpha$ & 4 \\
Time-scale separation of & & \\
carrier and photon lifetimes & $T$ & 200 \\
Injection current & $p$ & 0.1 \\
Coupling strength & $\sigma$ & 0.12 \\
Coupling delay time & $\tau$ & 100 \\
\hline \hline
\end{tabular}

A network of coupled lasers modeled by the LangKobayashi equations can be written in the form of Eq. (1), where $x^{i}(t)=\left(n^{i}, \operatorname{Re} E^{i}, \operatorname{Im} E^{i}\right)$ is now three dimensional and contains the real and imaginary part of the electric field $E^{i}$ and the charge carrier inversion $n^{i}$ of the $i$ th laser. A single laser is not chaotic, but the delayed feedback and/or coupling renders the system chaotic. The linear coupling function $H$ is represented by the matrix $\mathbf{H}=\left(\begin{array}{lll}0 & 0 & 0 \\ 0 & 1 & 0 \\ 0 & 0 & 1\end{array}\right)$, corresponding to all-optical coupling as in Eq. (17).

We consider a pair of lasers with overall coupling strength $\sigma$ and self-feedback strength $\kappa$. That is, the coupling matrix is given by

$$
G=\left[\begin{array}{cc}
\kappa & 1-\kappa \\
1-\kappa & \kappa
\end{array}\right] .
$$

Note that in the SM, the trajectory Eq. (2) does not depend on the parameter $\kappa$. Similar to the case of the maps discussed before, the spectrum of LEs is obtained from a Gram-Schmidt orthonormalization procedure, according to Farmer [14]. From this spectrum, we obtain the KY dimension using Eq. (5). Figure 9(a) shows $D_{K Y}$ as a function of $\kappa$. The dashed blue curve was obtained using the complete spectrum, while the solid green curve uses only the spectrum inside the SM. The vertical gray lines denote the boundary of stable synchronization of the two lasers with the given parameters; synchronization is stable between the two lines.

Outside the synchronization region, the complete spectrum has to be used when computing the KY dimension. However, for chaos synchronization, only the spectrum in the SM, given by Eq. (2), needs to be used. Thus, at the transition, the KY dimension has to jump from a high (dashed blue curve) to a lower value (solid green curve) for the SM.

Unfortunately, we are not able to calculate the correlation dimension of the laser rate equations. Due to the delay term which makes the system high dimensional, the available algorithms do not produce reliable results, to our knowledge.

We have also calculated the Kolmogorov-Sinai entropy $K$. It is defined as the sum of all positive LEs. Figure 9(b) shows the result $K(\kappa)$ for the pair of lasers, as before. Again, the dashed blue curve was obtained using the complete spectrum, while the solid green curve uses only the spectrum inside the SM. At the transition, the derivative of $K(\kappa)$ is discontinuous. In the desynchronized region, the second band of LEs crosses zero, as shown in Figs. 1 and 3 for the Bernoulli and the tent map triangle, respectively, and therefore it contributes to the Kolmogorov-Sinai entropy. 

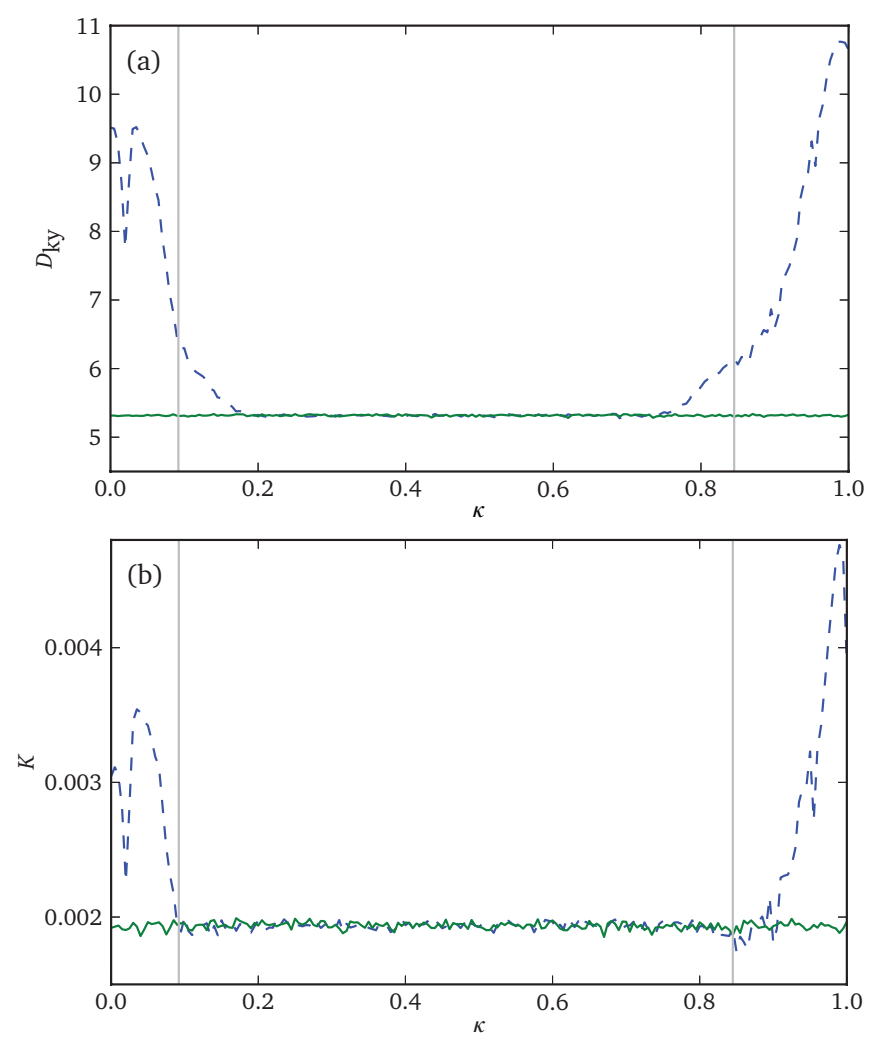

FIG. 9. (Color online) (a) Kaplan-Yorke dimension $D_{K Y}$ and (b) Kolmogorov-Sinai entropy $K$ of two coupled semiconductor lasers in dependence on the relative self-feedback strength $\kappa$; cf. Eq. (18). The dashed blue and solid green lines are obtained using the complete spectrum and the spectrum inside the SM only, respectively.

\section{NETWORKS}

In this section, we investigate the transition to chaos synchronization in large networks. For simplicity, we consider Bernoulli networks of $N$ units with all-to-all couplings without self-feedback. The coupling matrix $G$ of Eq. (7) has the eigenvalues $\gamma_{1}=1$ and $\gamma_{j}=-1 /(N-1), 1<j \leqslant N$.

Figure 10 shows the spectrum of LEs as a function of $\epsilon$ for a system of five all-to-all coupled Bernoulli units. Since the eigenvalue corresponding to the transversal spectrum has the multiplicity four, this spectrum is fourfold degenerated. For $\epsilon=0$, the uncoupled Bernoulli units have the LE $\lambda=\log a$. Hence, the KY dimension is $D_{K Y}=N$, i.e., the full phase space. As discussed before, at the transition to synchronization, only the $\gamma_{1}$ band contributes to $D_{K Y}$, hence the KY dimension jumps at the transition. The entropy has a kink, since at the transition the $N-1$ many $\gamma_{j}$ bands no longer contribute to the entropy.

We consider the limit of large delay times, $\tau \rightarrow \infty$. In this limit, using Eq. (10), we find the critical value at which the transition to synchronization occurs to be $\epsilon_{c}=(a-1) /[a(1-$ $\left.\left.\left|\gamma_{j}\right|\right)\right]$. For a coupling strength $\epsilon<1-1 / a$, the system is in the regime of strong chaos, otherwise it is in the regime of weak chaos [8]. In general, the transition from strong to weak chaos does not coincide with the synchronization transition, which usually occurs at larger values $\epsilon_{c} \geqslant 1-1 / a$; see Eq. (4). Only in the limit of $N \rightarrow \infty$ do both transitions fall together. The

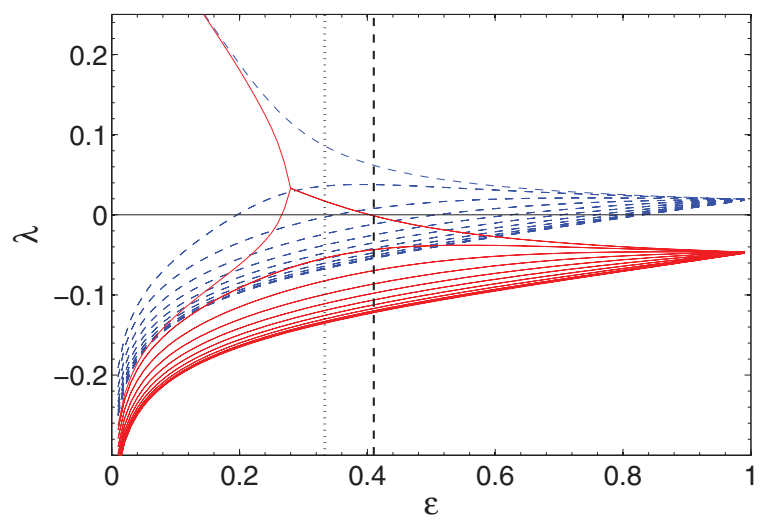

FIG. 10. (Color online) Lyapunov spectra vs coupling strength $\epsilon$ for a system of five all-to-all coupled Bernoulli maps with the parameters $a=1.5$ and $\tau=20$. The blue dashed lines show the longitudinal spectrum associated with $\gamma_{1}=1$ and the red solid lines show the transversal spectrum associated with the fourfold degenerated eigenvalue $\gamma_{2}=-1 / 4$. The vertical dotted line indicates the transition between strong and weak chaos and the vertical dashed line indicates $\epsilon_{c}$ where to its right the system is synchronized.

transition from strong to weak chaos is defined by the change in sign of the so-called local Lyapunov exponent [8]. For strong chaos, it is positive, and for weak chaos, it is negative.
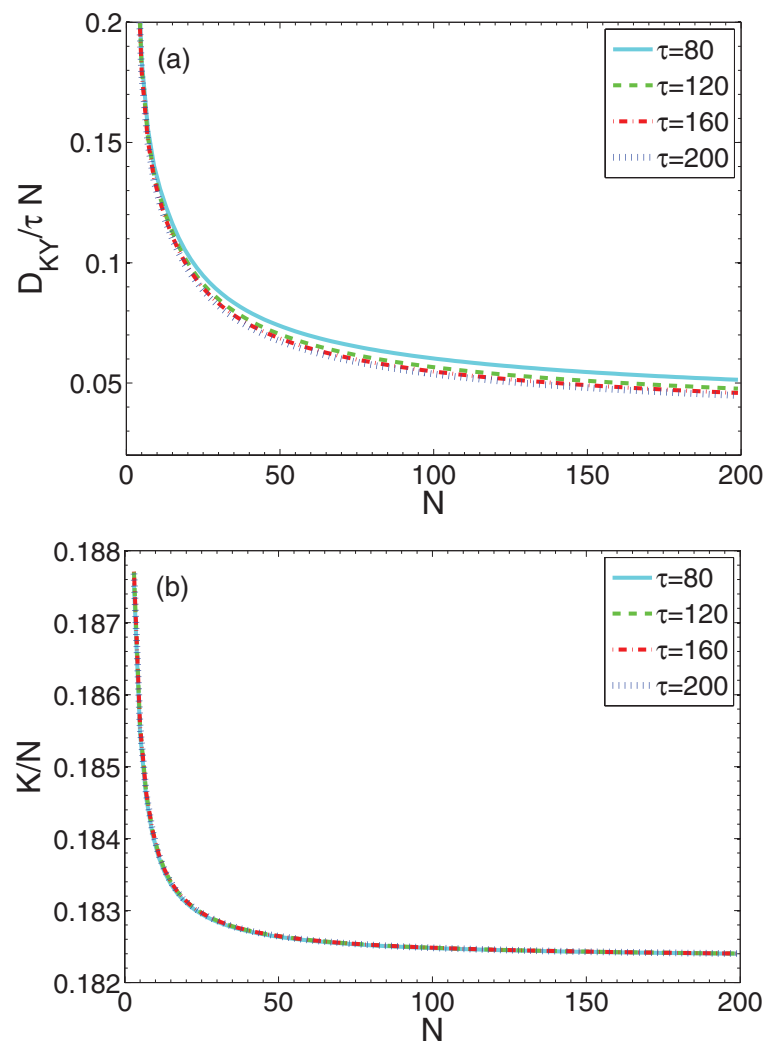

FIG. 11. (Color online) (a) KY dimension $D_{K Y}$ and (b) Kolmogorov entropy $K$ with respect to the number of all-to-all coupled Bernoulli maps $N$ for different delay times $\tau$. It starts from $\tau=80$ (uppermost curve) and increases in steps of $\Delta \tau=40$ up to $\tau=200$ (lowermost curve). Note that the curves (nearly) coincide. The other parameters are $a=1.5$ and $\epsilon=0.2$. 
For strong chaos, the maximum LE is of the order of one, whereas for weak chaos, it scales as $1 / \tau$. Since each mode of the network has $\tau$ many LEs of the order of $1 / \tau$, the KY dimension increases linearly with the delay time $\tau$, whereas the Kolmogorov entropy $K$ is independent of $\tau$. For a large enough coupling strength $\epsilon$, such that the system is synchronized, the dimension is determined solely by the SM and therefore the dimension is independent of the number of units $N$ and cannot exceed a value larger than the delay time of a single unit.

Figure 11 shows the KY dimension and the Kolmogorov entropy as a function of the system size $N$ in the regime of strong chaos for different delay times $\tau$. It is clearly visible that for large delay times, the different plots of $D_{K Y} / \tau N$
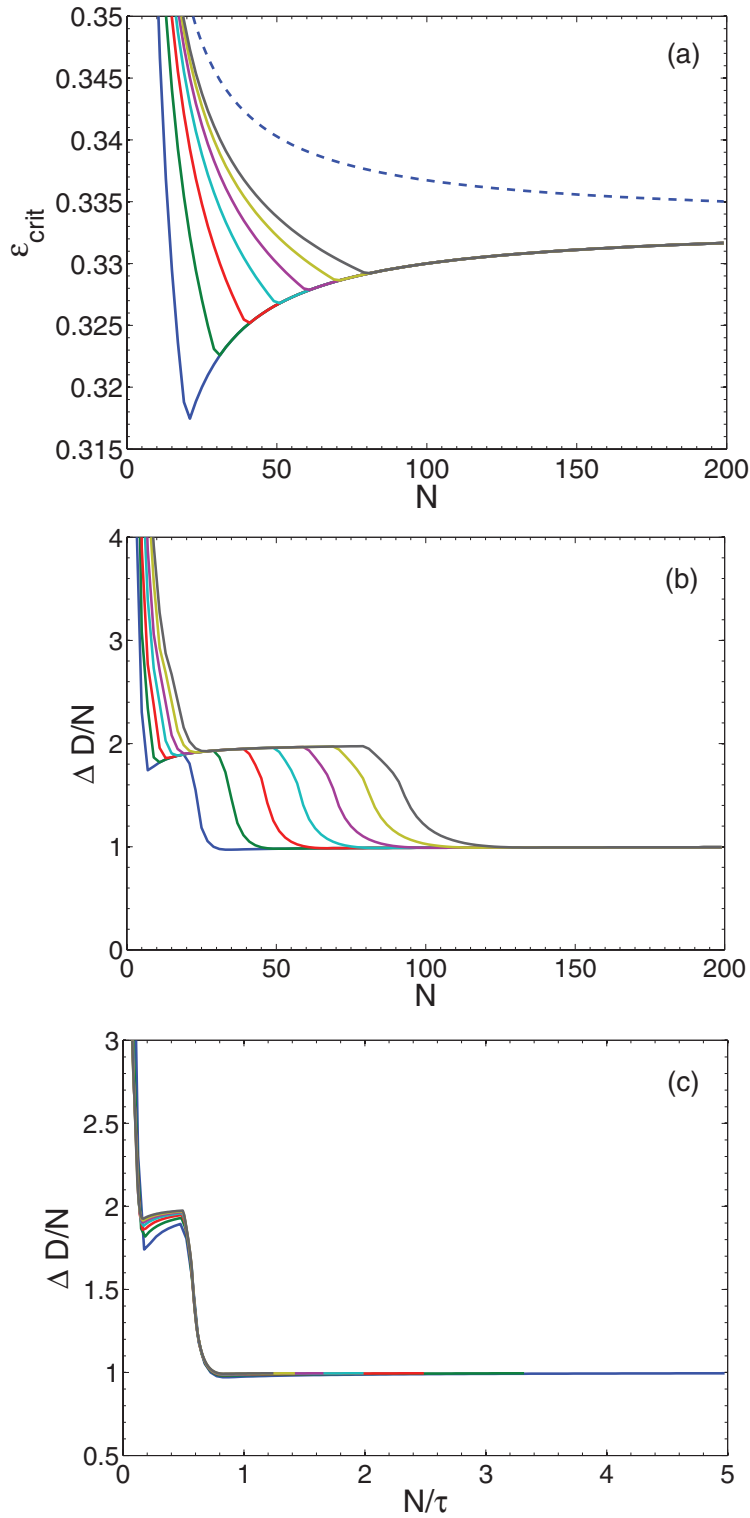

FIG. 12. (Color online) (a) Critical coupling strength $\epsilon_{c}$ and (b), (c) jump of the KY dimension $\Delta D_{K Y}$ with respect to the system size $N$ of all-to-all coupled Bernoulli maps. The different curves correspond to different delay times $\tau$. The lowest curve is for $\tau=40$ and increases by steps of $\Delta \tau=20$ up to $\tau=160$ (upper curve). For $\epsilon_{c}$, the theoretical value in the limit of $\tau \rightarrow \infty$ is plotted as well (upper dashed line). The parameter of the Bernoulli map is $a=1.5$. corresponding to different delays nearly coincide. Thus the KY dimension scales linearly with $\tau$, whereas the Kolmogorov entropy is independent of $\tau$. Both entropy as well as dimension increase with system size $N$ and it seems that for large $N$, both quantities scale linearly with $N$.

As discussed before, the transition to synchronization occurs at $\epsilon_{c}$, which depends on the number of units in the network. In the limit of large delay times $\tau \rightarrow \infty$, we find $\epsilon_{c}=$ $(a-1) /\left[a\left(1-\left|\gamma_{j}\right|\right)\right]$. Thus $\epsilon_{c}$ decreases monotonically towards $\epsilon=1-1 / a$ for $N \rightarrow \infty$. At the transition, only the $\gamma_{1}$ band contributes to $D_{K Y}$ and hence the KY dimension jumps.

Figure 12 shows the jump of the KY dimension at the transition as well as the critical coupling strength $\epsilon_{c}$ at which the jump occurs as a function of network size $N$ for different delay times. Since $\epsilon_{c}$ depends on $N$, the jump has a nonmonotonic behavior. For large enough network sizes, the jump $\Delta D$ scales linearly with the number of units. The slope of this linear relation is approximately two for small $N$, $\Delta D / N \approx 2$, and approximately one for large $N, \Delta D / N \approx 1$. The transition between the two different slopes is related to the dip in the critical coupling strength $\epsilon_{c}(N)$, which is due to the fact that the system is not yet in the limit $\tau \rightarrow \infty$.

The slope $\Delta D / N$ depends on the order of limits. If we take the limit $\tau \rightarrow \infty$ first, the jump $\Delta D$ scales linearly with the number of units $N$ with a slope of two. If we, however, take the limit $N \rightarrow \infty$ first, it scales with a slope of one for any value of $\tau$.

In Fig. 12(c), the same data as in Fig. 12(b) are plotted as $\Delta D / N$ versus $N / \tau$, leading to data collapse. This shows that the jump in the Kaplan-Yorke dimension is in the limit of large $\tau$ determined by the scaling law

$$
\Delta D \approx N \psi(N / \tau),
$$

where $\psi$ is the scaling function depicted in Fig. 12(c).

\section{SUMMARY AND DISCUSSION}

Networks of identical nonlinear units can synchronize to a common chaotic trajectory. Although the time delay of the coupling can be very large, the units can synchronize without any time shift. We investigated the transition to chaos synchronization in the limit of very large delay times. General arguments about the dynamic of such a network predict a jump of the KY dimension of the chaotic attractor when the network synchronizes. In addition, the Kolmogorov prediction entropy should show a discontinuous slope.

We tested these general predictions for networks of iterated maps. For Bernoulli maps, our numerical results show a clear discontinuous behavior of the attractor dimension. The KY as well as the correlation dimension jump to a low value when the network synchronizes. For tent maps, the numerical results of the correlation dimension are not so clear due to large statistical fluctuations caused by limited computational power. Nevertheless, our results indicate a jump in the attractor dimension, too. In both cases, the prediction entropy shows a discontinuous slope.

The KY dimension was also calculated for the rate equation of semiconductor lasers. Again, our general arguments give a discontinuous KY dimension at the transition. Unfortunately, 
we were not able to calculate the correlation dimension for this case.

For Bernoulli networks, we numerically calculated the KY dimension as a function of system size $N$ and delay time $\tau$ in the region of strong chaos. The dimension scales with $N \tau$. The jump of the dimension at the transition scales with $N$, as well.

Our results show that one has to use the standard definition of the KY dimension, given by Eq. (5), with care. If we use Eq. (5) with all possible LEs, we obtain a wrong result. Here we argued that not all LEs contribute to Eq. (5) in the case of synchronization. This argument predicts a jump in the attractor dimension, which is in agreement with our numerical results of the correlation dimension. One may argue that the KY dimension describes the dimension of typical attractors only [17]. In fact, the SM is sensitive to detuning the units, which points towards a nontypical attractor. On the other side, our results show that the corrected KY dimension is close to the correlation dimension, which is well defined for the SM.
Hence, it seems that the corrected KY dimension describes the SM correctly.

In general, it may not be obvious which LEs contribute to the KY dimension. For example, if we distort the SM by a nonlinear transformation of one unit, the dimension does not change but we do not know which LEs have to be omitted in this case. Also, in the case of generalized synchronization, the dynamics is restricted to a low-dimensional manifold which rules out the majority of LEs. But, again, we do not know in advance which LEs have to be omitted from Eq. (5).

Our argument of omitting bands of negative LEs relies on the fact that the dynamics is restricted to the SM. However, a tiny detuning of the nonlinear units leads to imperfect synchronization and this argument is no longer valid. Thus, the attractor dimension should immediately jump to a high value for any amount of detuning. We have tried to calculate the dimension in the limit of zero detuning, but our numerical results did not allow a conclusive statement.
[1] L. M. Pecora and T. L. Carroll, Phys. Rev. Lett. 64, 821 (1990). [2] A. S. Pikovsky, Z. Phys. B 55, 149 (1984).

[3] S. Boccaletti, J. Kurths, G. Osipov, D. Valladares, and C. Zhou, Phys. Rep. 366, 1 (2002).

[4] S. Boccaletti, V. Latora, Y. Moreno, M. Chavez, and D.-U. Hwang, Phys. Rep. 424, 175 (2006).

[5] R. Albert and A.-L. Barabási, Rev. Mod. Phys. 74, 47 (2002).

[6] A. Arenas, A. Díaz-Guilera, J. Kurths, Y. Moreno, and C. Zhou, Phys. Rep. 469, 93 (2008).

[7] E. Klein, N. Gross, M. Rosenbluh, W. Kinzel, L. Khaykovich, and I. Kanter, Phys. Rev. E 73, 066214 (2006).

[8] S. Heiligenthal, T. Dahms, S. Yanchuk, T. Jüngling, V. Flunkert, I. Kanter, E. Schöll, and W. Kinzel, Phys. Rev. Lett. 107, 234102 (2011).

[9] F. M. Atay, J. Jost, and A. Wende, Phys. Rev. Lett. 92, 144101 (2004).

[10] A. C. Marti, M. Ponce, and C. Masoller, Phys. Rev. E 72, 066217 (2005).

[11] C. Masoller and A. C. Marti, Phys. Rev. Lett. 94, 134102 (2005).

[12] M. Zigzag, M. Butkovski, A. Englert, W. Kinzel, and I. Kanter, Phys. Rev. E 81, 036215 (2010).

[13] S. Lepri, G. Giacomelli, A. Politi, and F. T. Arecchi, Physica D (Amsterdam, Neth.) 70, 235 (1994).

[14] J. D. Farmer, Physica D (Amsterdam, Neth.) 4, 366 (1982).

[15] M. Escalona-Morán, G. Paredes, and M. G. Cosenza, Int. J. App. Math. Stat. 26, 58 (2012).

[16] A. Pikovsky, M. Rosenblum, and J. Kurths, Synchronization: A Universal Concept in Nonlinear Sciences (Cambridge University Press, Cambridge, UK, 2001).
[17] J. Farmer, E. Ott, and J. A. Yorke, Physica D (Amsterdam, Neth.) 7, 153 (1983).

[18] J. Kaplan and J. Yorke, in Functional Differential Equations and Approximation of Fixed Points, edited by H. O. Peitgen and H. O. Walther (Springer, Heidelberg/New York, 1979).

[19] P. Grassberger and I. Procaccia, Phys. Rev. Lett. 50, 346 (1983).

[20] P. Frederickson, J. L. Kaplan, E. D. Yorke, and J. A. Yorke, J. Differ. Equations 49, 185 (1983).

[21] J. Argyris, G. Faust, M. Haase, and R. Friedrich, Die Erforschung des Chaos, 2nd ed. (Springer, Heidelberg/New York, 2010).

[22] P. Grassberger and I. Procaccia, Physica D (Amsterdam, Neth.) 9, 189 (1983).

[23] P. Grassberger and I. Procaccia, Physica D (Amsterdam, Neth.) 13, 34 (1984).

[24] L. M. Pecora and T. L. Carroll, Phys. Rev. Lett. 80, 2109 (1998).

[25] V. Flunkert, S. Yanchuk, T. Dahms, and E. Schöll, Phys. Rev. Lett. 105, 254101 (2010).

[26] H. G. Schuster and W. Just, Deterministic Chaos: An Introduction, 4th ed. (Wiley, New York, 2005).

[27] A. Englert, S. Heiligenthal, W. Kinzel, and I. Kanter, Phys. Rev. E 83, 046222 (2011).

[28] R. Hegger, H. Kantz, and T. Schreiber, Chaos 9, 413 (1999).

[29] H. Kantz and T. Schreiber, Nonlinear Time Series Analysis, 2nd ed. (Cambridge University Press, Cambridge, UK, 2005).

[30] M. Lakshmanan and D. Senthilkumar, Dynamics of Nonlinear Time-Delay Systems, Springer Series in Synergetics (Springer, Berlin, 2011).

[31] R. Lang and K. Kobayashi, IEEE J. Quantum Electron. 16, 347 (1980). 